\title{
Simvastatin and lovastatin inhibit breast cell invasion induced by H-Ras
}

\author{
SOOUK KANG, EUN-SOOK KIM and AREE MOON \\ College of Pharmacy, Duksung Women's University, Seoul 132-714, Korea
}

Received December 23, 2008; Accepted February 5, 2009

DOI: $10.3892 /$ or_00000357

\begin{abstract}
Breast cancer mortality is strongly related to the invasive and metastatic potential of tumor cells. We previously showed that an active mutant of $\mathrm{H}$-Ras induced invasive phenotype of MCF10A human breast epithelial cells. Membrane anchoring of Ras requires isoprenylation which involves the activity of 3-hydroxy 3-methylglutaryl (HMG)CoA reductase. In this study, we investigated the inhibitory effect of HMG-CoA reductase inhibitors, widely used for hypercholesterolemia, on H-Ras-induced invasion of MCF10A cells. Treatment of H-Ras MCF10A cells with simvastatin and lovastatin markedly decreased isoprenylated H-Ras in membrane fraction while the unprenylated H-Ras was increased in cytosol fraction, demonstrating that these statins inhibited membrane anchoring of H-Ras in MCF10A cells. Simvastatin and lovastatin significantly inhibited H-Rasinduced invasion which was reversed by farnesyl pyrophosphate (FPP), indicating that the inhibitory effect was related to inhibition of the biosynthesis of prenylated derivatives. Statins downregulated matrix metalloproteinase (MMP)-9 and, to a lesser extent, MMP-2 in H-Ras MCF10A cells. Simvastatin and lovastatin inactivated H-Ras downstream signaling molecules, possibly by inhibiting H-Ras membrane localization and thus its function in MCF10A cells. Taken together, this study clearly demonstrated the inhibitory effect of simvastatin and lovastatin on H-Ras-induced invasion, MMP expression and signal transduction in MCF10A breast epithelial cells, providing supporting rationale for future statin trials as a therapeutic intervention to regulate breast cancer metastasis.
\end{abstract}

\section{Introduction}

Breast cancer is estimated as one of the most commonly diagnosed types of cancer among women and metastasis is the principal cause of death from breast cancer. Elevated levels of Ras expression are often associated with tumor aggressiveness

Correspondence to: Dr Aree Moon, College of Pharmacy, Duksung Women's University, Seoul 132-714, Korea

E-mail: armoon@duksung.ac.kr

Key words: H-Ras, 3-hydroxy 3-methylglutaryl-CoA reductase, breast cell invasion, matrix metalloproteinase- 2 in breast cancer, including the degrees of invasion to fat tissues and infiltration into lymphatic vessels (1-3). It has been reported that a single point mutation at amino acid residue 12 (Gly to Asp) of H-Ras is often found in mammary carcinoma (4). We previously showed that active mutant $\left(\right.$ Gly $^{12}$ to $\mathrm{Asp}^{12}$ ) of H-Ras induced invasive and migratory phenotypes of MCF10A human breast epithelial cells $(5,6)$.

For effective Ras signaling, attachment of Ras proteins to plasma membrane is required. Ras proteins undergo C-terminal lipid modification, isoprenylation, which is essential for their proper membrane anchoring and function (7). H-Ras undergoes farnesylation exclusively while K-Ras and N-Ras can undergo alternative prenylation, geranylgeranylation (8). Isoprenylation of Ras involves the activity of 3-hydroxy 3-methylglutaryl (HMG)-CoA reductase, a key enzyme for mevalonate synthesis which is the precursor molecule for the generation of farnesylpyrophosphate (FPP) and geranylgeranylpyrophosphate (GGPP). HMG-CoA reductase inhibitors called statins have been shown to reduce intracellular isoprenoids pools such as FPP or GGPP and thus are speculated to modulate cellular functions of Ras proteins by inhibiting their membrane localization (9).

There is an expanding body of evidence supporting the ability of statins to exert anti-cancer effects on various types of human cancer cells (10). In experimental models, statin treatment was shown to diminish the growth of fibrosarcomas and experimental lung metastases in rats (11). Moreover, in a major clinical trial, simvastatin was shown to have cancer prevention capabilities (12). Atorvastatin markedly enhanced the inhibitory effect of anti-cancer drugs on cell migration and invasion in vitro and suggested improvement of clinical presentations $(13,14)$. It prevented isoprenylation of RhoA in human melanoma cells and inhibited metastasis in vivo $(15,16)$. Lovastatin and cerivastatin have been shown to inhibit metastatic potential of breast cancer cells $(17,18)$. Cerivastatin, however, has been withdrawn from the market, due to studies of fatal rhabdomyolysis (19).

In the present study, we evaluated the ability of simvastatin and lovastatin, commonly used drugs for the treatment of hypercholesterolemia, to inhibit the H-Ras-induced invasiveness in MCF10A human breast epithelial cells. We show that these statins inhibited membrane anchoring of H-Ras as well as H-Ras-induced invasive phenotype in MCF10A cells. Since breast cancer mortality is strongly related to the capacity of tumor cells to invade and metastasize and active mutation of H-Ras is associated with tumor aggressiveness in breast cancer, our findings may provide supporting rationale for 
future statin trials in breast cancer patients as new therapeutical approaches with the association of chemotherapy.

\section{Materials and methods}

Cell lines. Development and characterization of H-Ras MCF10A cells were described previously (5). The cells were cultured in DMEM/F12 medium supplemented with 5\% horse serum, $0.5 \mu \mathrm{g} / \mathrm{ml}$ hydrocortisone, $10 \mu \mathrm{g} / \mathrm{ml}$ insulin, $20 \mathrm{ng} / \mathrm{ml}$ epidermal growth factor, $0.1 \mu \mathrm{g} / \mathrm{ml}$ cholera enterotoxin, $100 \mathrm{U} / \mathrm{ml}$ penicillin-streptomycin, $2 \mathrm{mM}$ L-glutamine and $0.5 \mu \mathrm{g} / \mathrm{ml}$ amphotericin B.

Cell fractionation. Cells were treated with simvastatin or lovastatin for $24 \mathrm{~h}$. At $~ 80 \%$ confluency, growth medium was removed, cells were rinsed with balanced salt solution and homogenized. After whole fraction was collected, the disrupted cells were centrifuged for $10 \mathrm{~min}$ at $1,000 \mathrm{x} \mathrm{g}$ and $4^{\circ} \mathrm{C}$ resulting in the pellet and supernatant. The supernatant was centrifuged for $50 \mathrm{~min}$ at $30,000 \mathrm{x} \mathrm{g}$ and $4^{\circ} \mathrm{C}$, resulting in the supernatant (cytosolic fraction) and the pellet. Following the suspension in chelating buffer, pellet was centrifuged at 30,000 x $\mathrm{g}$ for $30 \mathrm{~min}$. The supernatant was discarded and the pellet was washed in incubation buffer and spun down at 30,000 x g. The resulting pellet (membrane fraction) was resuspended in incubation buffer.

Immunoblot analysis. Immunoblot analysis was performed as previously described (6). Anti-p38 MAPK, anti-phosphorylated p38 MAPK, anti-ERK1/2, anti-phosphorylated ERK1/2, antiphosphorylated MEK1/2, anti-Raf-1, anti-phosphorylated Raf- 1 and $B$-actin antibodies were purchased from Cell Signaling Tech (Beverly, MA). MMP-2 antibody was purchased from R\&D systems (Minneapolis, MN). H-Ras, PI3K, phosphor-tyrosine and MMP-9 antibodies were purchased from Santa Cruz Biotech (Santa Cruz, CA).

Raf-1 activity assay. The Raf-1 activity was performed as previously described (6). The lysate was preincubated with protein A-Sepharose suspension (Roche Applied Science). The supernatant was incubated with anti-Raf-1 antibody. Protein A-Sepharose suspension was added and incubation was extended overnight on ice with shaking. The antibodyantigen complex was washed twice in lysis buffer and twice in high salt buffer and then the SDS-PAGE loading buffer was added. Immunoblot analysis was performed to assess the activity of immunoprecipitated Raf-1 with anti-phosphorylated Raf-1 antibody.

PI3K activity assay. The PI3K activity was determined as previously described (20). Briefly, cell lysates were centrifuged for $15 \mathrm{~min}$ at $12,000 \mathrm{x} \mathrm{g}$ to remove debris, immunoprecipitated using anti-phosphotyrosine antibody and immobilized protein G-agarose beads (Pierce) to the antigen-antibody complex, and incubated for $2 \mathrm{~h}$ at $4^{\circ} \mathrm{C}$. Immunoprecipitates were washed three times with radioimmune precipitation buffer and denatured in Laemmli sample buffer. The supernatants were resolved by $8 \%$ reducing SDS-PAGE. Tyrosinephosphorylated PI3K proteins were detected by immunoblotting using anti-PI3K antibody.

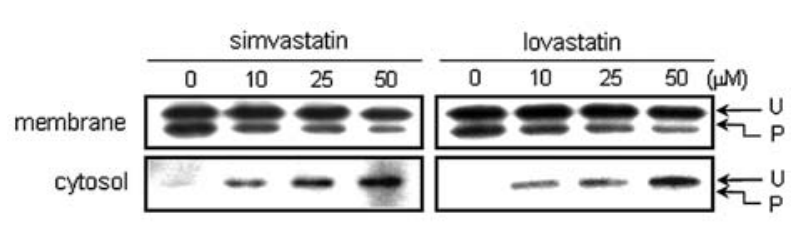

Figure 1. Simvastatin and lovastatin inhibited membrane anchoring of H-Ras. H-Ras MCF10A cells were fractionated as previously described in Materials and methods after treated with simvastatin or lovastatin for indicated concentration for $24 \mathrm{~h}$. The localization of Ras in two fractions (cytosol and membrane) were determined by immunoblot analysis.

Detection of Rho-GTP, Racl-GTP and Ras-GTP. For the Rho GTPase activity, cells were washed with ice-cold PBS and lysed in MLB buffer. Cell lysates were clarified by centrifugation and incubated with GST-RBD bound to glutathione-sepharose beads (Upstate, Charlottesville, VA). The beads were washed four times with MLB buffer and bound Rho proteins were detected by immunoblot with an antibody against Rho (Upstate).

The level of Rac1-GTP was measured by affinity precipitation using PAK-1 p21-binding domain Rac assay reagent (Upstate) following the manufacturer's instruction, as previously described (20). The level of Ras-GTP was measured by affinity precipitation using Raf-1 RBD (human Ras binding domain, residues 1 to 149 ) reagent (Upstate) following the manufacturer's instruction.

Gelatin zymogram assay. Cells were cultured in serum-free DMEM/F12 medium for $48 \mathrm{~h}$. Gelatinolytic activity of the conditioned medium was determined by gelatin zymogram assay, as previously described (5). Areas of gelatinase activity were detected as clear bands against the blue-stained gelatin background.

In vitro invasion assay. In vitro invasion assay was performed using 24-well Transwell unit with polycarbonate filters (Corning Costar, Cambridge, MA), as previously described (5).

In vitro migration assay using Transwell. In vitro migration assay was performed using a 24-well Transwell unit with polycarbonate filters, as previously described (6).

Densitometry measurements. Relative band intensities were determined by quantitation of each band with an image analyzer (Vilber Lourmat, Marne-la-Cedex 1, France).

\section{Results}

Simvastatin and lovastatin inhibited membrane anchoring of H-Ras. To examine if simvastatin and lovastatin inhibit membrane anchoring of H-Ras protein, H-Ras MCF10A cells were treated with various concentrations of these statins and were fractionated to obtain the membrane and cytosolic fractions. In membrane fraction, control cells showed comparable amounts of both unprenylated and prenylated forms of H-Ras (Fig. 1). Treatment of H-Ras MCF10A cells with simvastatin or lovastatin markedly reduced the level of prenylated H-Ras in membrane fraction in a dose-dependent 
A

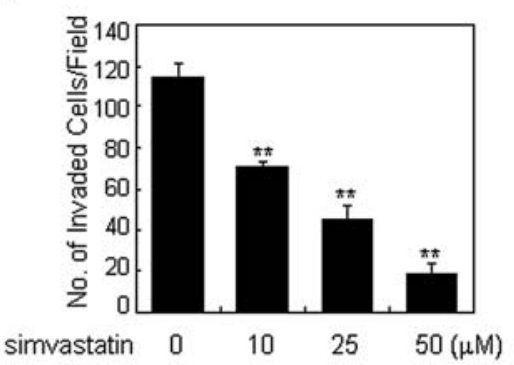

B

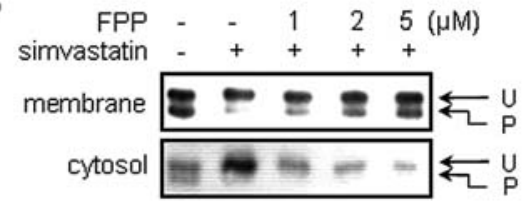

C

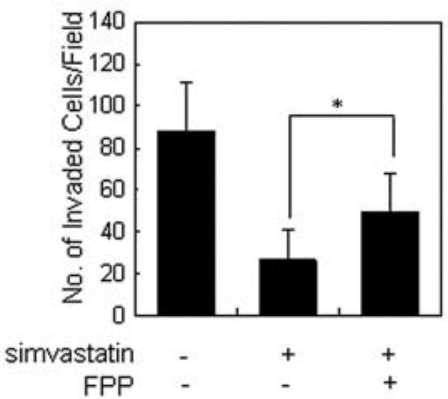

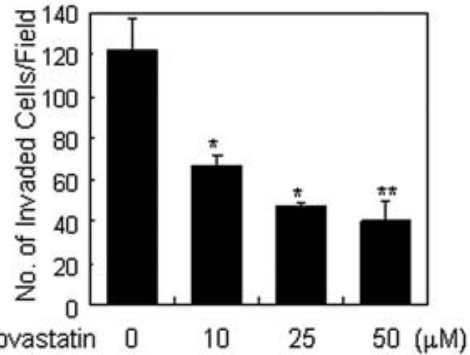
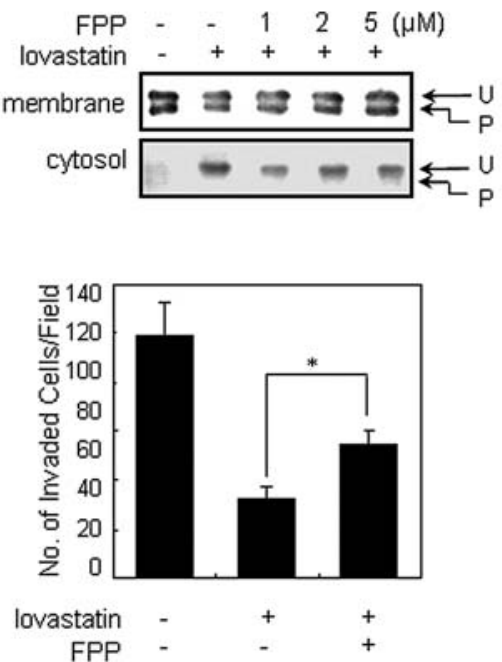

Figure 2. Statins inhibit H-Ras-induced invasion which is reversed by FPP. (A) H-Ras MCF10A cells were performed Transwell invasion assay treated with simvastatin or lovastatin for indicated concentration for $17 \mathrm{~h}$. The number of invaded cells per field was counted (x400) in thirteen fields. The results represent mean \pm SE of triplicates. ${ }^{*},{ }^{*}$ Statistically different from control at $\mathrm{p}<0.05$ and $\mathrm{p}<0.01$, respectively, by the two-tailed Student's t-test. (B) H-Ras MCF10A cells were treated with $50 \mu \mathrm{M}$ simvastatin and lovastatin with or without FPP for indicated concentration for $24 \mathrm{~h}$. The localization of Ras in two fractions (cytosol and membrane) were determined by immunoblot analysis. (C) H-Ras MCF10A cells were treated with $50 \mu \mathrm{M}$ simvastatin and lovastatin with or without $10 \mu \mathrm{M}$ FPP for $17 \mathrm{~h}$. The number of invaded cells per field was counted (x400) in thirteen fields. The results represent mean \pm SE of triplicates. ${ }^{*}$ Statistically different from control at $\mathrm{p}<0.05$ by the two-tailed Student's t-test.

manner. Unprenylated H-Ras was only slightly decreased by the statin treatment. Simvastatin and lovastatin dosedependently increased unprenylated H-Ras in the cytosolic fraction, indicating that the statins inhibited membrane localization of H-Ras protein by preventing isoprenylation of H-Ras.

Statins inhibit H-Ras-induced invasion which is reversed by FPP. We then investigated the effect of statins on invasive capacity of H-Ras MCF10A cells which are originally highly invasive. Treatment with simvastatin or lovastatin significantly inhibited the invasion of $\mathrm{H}$-Ras MCF10A cells in a dosedependent manner (Fig. 2A). To address if the inhibition of $\mathrm{H}$-Ras MCF10A cell invasion by simvastatin and lovastatin was due to inhibition of the biosynthesis of prenylated derivatives, H-Ras MCF10A cells were treated simvastatin or lovastatin in the absence or presence of FPP. Decreased level of prenylated $\mathrm{H}$-Ras in the membrane fraction of $\mathrm{H}$-Ras MCF10A cells treated with the statins was dose-dependently restored by FPP (Fig. 2B). As shown in Fig. 2C, $50 \mu \mathrm{M}$ of FPP significantly reversed simvastatin- and lovastatin-inhibited invasion H-Ras MCF10A cells. The data demonstrated that the inhibitory effect of simvastatin and lovastatin on invasiveness of H-Ras MCF10A cells was related to inhibition of the biosynthesis of prenylated derivatives.

Statins downregulate MMP-9 and to a lesser extent, MMP-2 in H-Ras MCF10A cells. Since tumor cell invasion is often associated with enhanced synthesis of matrix metalloproteinase (MMP)-2 and/or MMP-9, we examined the effect of statins on the expression of these proteins. A marked inhibition of MMP-9 was observed in H-Ras MCF10A cells treated with simvastatin as evidenced by gelatin zymogram assay (Fig. 3A, top). At $10 \mu \mathrm{M}$ concentration of simvastatin, MMP-9 level was reduced by $75 \%$. MMP-2 level was also decreased by simvastatin, but to a lesser extent compared to MMP-9. These data were confirmed by Western blot analysis (Fig. 3A, bottom). Similar results were obtained when H-Ras MCF10A cells were treated with lovastatin (Fig. 3B). The results demonstrate that simvastatin and lovastatin dose-dependently downregulate MMP-9 and to a lesser extent, MMP-2 in H-Ras MCF10A cells.

Statins inhibited activation of H-Ras downstream signaling molecules. We previously showed that H-Ras activated Raf, MEK, ERK-1/2, Rac1, PI3K and p38 MAPK in MCF10A 
A
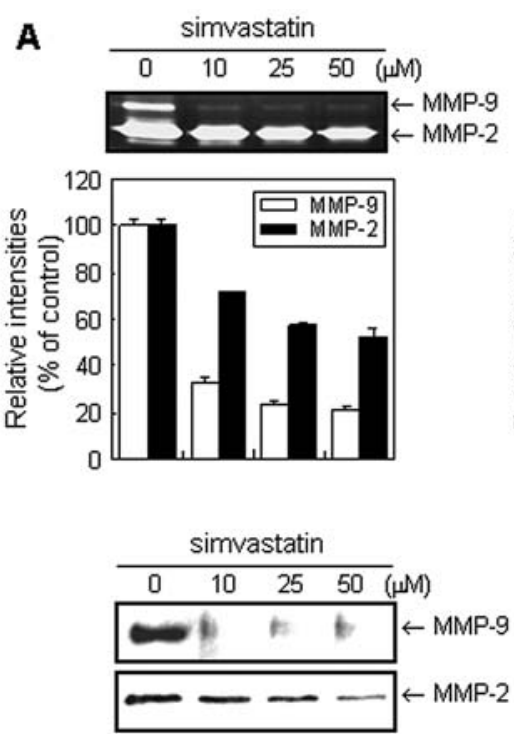

B
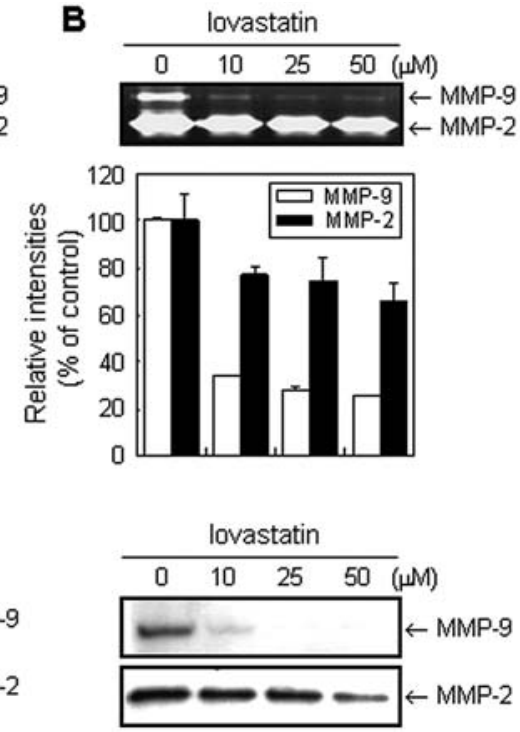
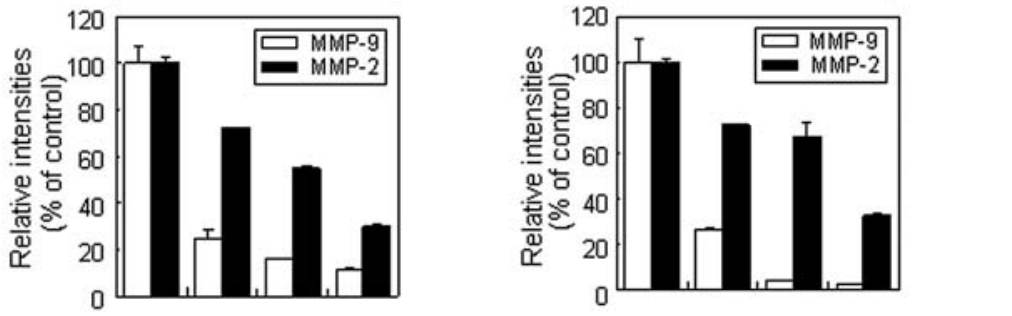

Figure 3. Statins downregulate MMP-9 and to a lesser extent, MMP-2 in H-Ras MCF10A cells. Gelatin zymogram assay was performed on the conditioned media of H-Ras MCF10A cells treated with $50 \mu \mathrm{M}$ of simvastatin (A) or lovastatin (B) for indicated concentration for 48 h. Expression of MMP-2 and MMP-9 were determined by immunoblot analyses of conditioned media using antibodies against MMP-2 and MMP-9. Relative band intensities were determined by densitometry measurements.

A
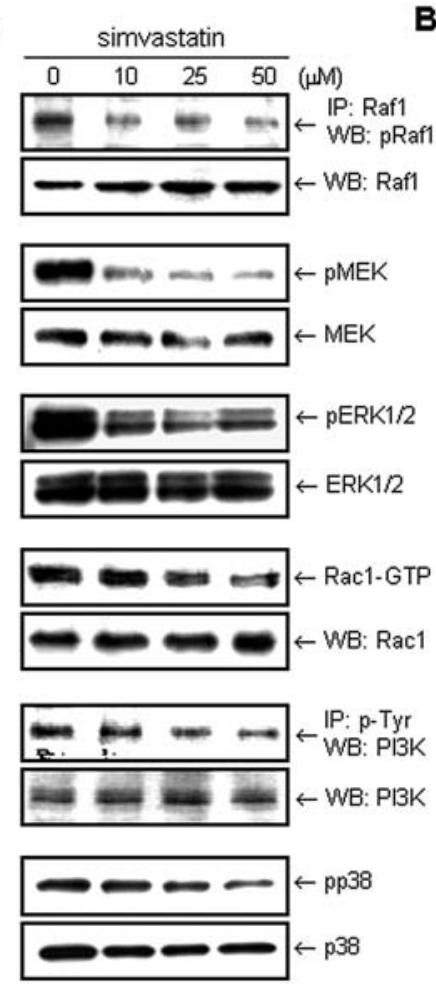

B
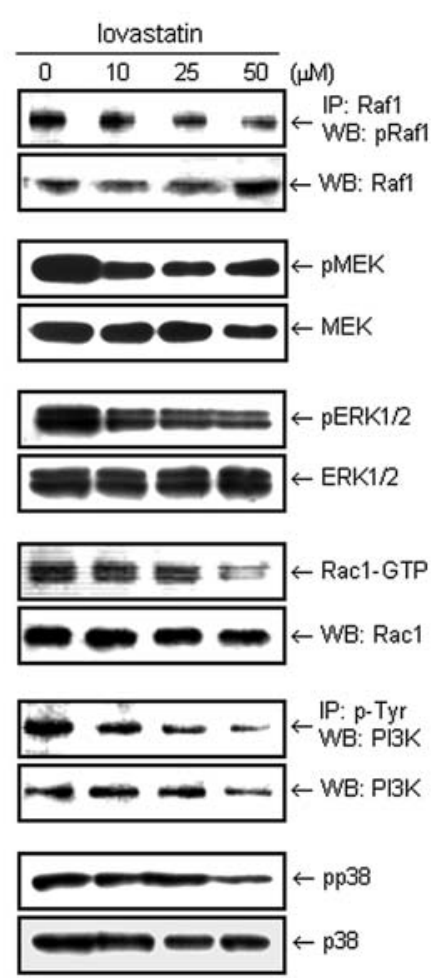

Figure 4. Statins inhibited activation of H-Ras downstream signaling molecules. H-Ras MCF10A cells were treated with simvastatin (A) and lovastatin (B) for indicated concentration for $24 \mathrm{~h}$. Cell lysates were immunoprecipitated with anti-Raf-1 antibody and probed with phosphorylated Raf-1 antibody. For the PI3K activity, cell lysates were immunoprecipitated with anti-phosphotyrosine antibody and the immunoprecipitates were subjected to immunoblot analysis with an anti-PI3K antibody. For the Rac1 activity, cell lysates were incubated with GST-p21-binding domain fusion protein and the bound active Rac1-GTP molecules were analyzed by immunoblotting using anti-Rac1 antibody. The levels of activated MEK, ERKs and p38 MAPK were determined by immunoblot analyses using phospho-specific antibodies (pMEK, pERKs and pp38, respectively). 
cells $(6,20)$. In order to elucidate the signaling pathways affected by statins, Western blot analysis was performed to detect the activated forms of signaling molecules in H-Ras MCF10A cells upon treatment with statins. As shown in Fig. 4A, simvastatin inhibited activation of signaling molecules Raf, MEK, ERK-1/2, Rac1, PI3K and p38 MAPK of H-Ras MCF10A cells in a dose-dependent manner. Lovastatin exerted the same effects on these signaling molecules (Fig. 4B). The results indicated that simvastatin and lovastatin prevented activation of $\mathrm{H}$-Ras downstream signaling molecules, possibly by inhibiting membrane localization of H-Ras in MCF10A cells.

\section{Discussion}

Since mortality from breast cancer is related to the capacity of tumor cells to invade and metastasize, strategies for therapeutic intervention to regulate breast cancer metastasis have been devised. Mutations or overexpression of the Ras oncogene are frequent genetic alterations in human tumors. We have previously demonstrated the involvement of H-Ras in invasive/ migratory potential of breast cells using MCF10A human breast epithelial cell system. In an approach to block the invasive ability of breast cells induced by $\mathrm{H}$-Ras, the present study investigated the inhibitory effect of statins on Ras membrane localization and metastatic properties of H-Ras MCF10A cells

HMG-CoA reductase inhibitors have been widely used for the treatment of hypercholesterolemia since mevalonate is a precursor of cholesterol synthesis $(21,22)$. Previously, it has been suggested that non-lipid-related effects of statins could play a beneficial role in cancer therapy, probably due to the inhibition of Ras and Rho (23). Breast cancer cells with activated Ras pathway seemed to be sensitive to breast cancer growth prevention by statin treatment (24).

Lovastatin inhibits experimental metastasis of mammary carcinoma cells through alterations in cytoskeletal organization (17). Cerivastatin, although it was withdrawn from the market, was shown to inhibit invasiveness and metastatic properties of highly invasive breast cancer cell line, MDA-MB-231, with spontaneous activation of Ras and $\mathrm{NF}_{\kappa} \mathrm{B}$ and overexpression of RhoA (18). Here, we report that simvastatin and lovastatin inhibited the invasive phenotype of H-Ras MCF10A cells. The inhibitory effect of simvastatin and lovastatin on invasiveness of H-Ras MCF10A cells was clearly related to inhibition of the biosynthesis of prenylated derivatives because it was shown to be reversed by FPP. H-Ras undergoes farnesylation exclusively while K-Ras and N-Ras can undergo alternative prenylation (geranylgeranylation) in the presence of farnesyltransferase inhibitors (FTIs) and therefore escape FTI-mediated inhibition of membrane association (8).

Members of the MMP family play a critical role in tumor invasion and metastasis formation, especially, MMP-2 and MMP-9 $(25,26)$. Simvastatin inhibits MMP-9 secretion by inhibiting RhoA pathway in human smooth muscle cells (27). The present study revealed that expression of MMP-9, and to a lesser extent MMP-2, was inhibited by lovastatin and simvastatin. Given the significant role of MMP-2 and MMP-9 in breast cell invasion, a detailed mechanism for the statininduced MMP-2/-9 downregulation needs to be further elucidated. Activation of Raf, MEK, ERK-1/2, Rac1, PI3K and p38 MAPK were inhibited by simvastatin and lovastatin in H-Ras MCF10A cells, suggesting that these signaling molecules may mediate the inhibitory effect of statins on breast cell invasion. Additional studies remain to be performed to further reveal the involvement of other signaling molecules in the inhibitory effect of simvastatin and lovastatin on H-Rasinduced invasive potential of MCF10A cells.

Taken together, the present study clearly demonstrated that simvastatin and lovastatin efficiently inhibited membrane localization of H-Ras and the invasive phenotype of MCF10A human breast epithelial cells induced by H-Ras. We also provided data suggesting the involvement of MMP-2/-9 in the inhibitory effect of statins on breast cell invasion. Given that breast cancer has been estimated as the most commonly diagnosed type of cancer among women and metastasis is the principal cause of death from breast cancer, our findings providing in vitro evidence for anti-invasive effect of simvastatin and lovastain on human breast epithelial cells may hold promise for future clinical studies.

\section{Acknowledgements}

This work was supported by the KOSEF NRL Program (MEST, No. ROA-2008-000-20070-0), KOSEF (MEST, No. R11-2007-107-01002-0), and by the KRF (KRF-2008-314C00230).

\section{References}

1. Clair T, Miller WR and Cho-Chung YS: Prognostic significance of the expression of a ras protein with a molecular weight of 21,000 by human breast cancer. Cancer Res 47: 5290-5293, 1987.

2. Clark GJ and Der CJ: Aberrant function of the Ras signal transduction pathway in human breast cancer. Breast Cancer Res Treat 35: 133-144, 1995 .

3. Watson DM, Elton RA, Jack WJ, Dixon JM, Chetty U and Miller WR: The H-ras oncogene product p21 and prognosis in human breast cancer. Breast Cancer Res Treat 17: 161-169, 1991.

4. Franks LM and Teich NM: Cellular and Molecular Biology of Cancer. Oxford University Press, 1997

5. Moon A, Kim MS, Kim TG, Kim SH, Kim HE, Chen YQ and Kim HR: H-ras, but not N-ras, induces an invasive phenotype in human breast epithelial cells: a role for MMP-2 in the H-rasinduced invasive phenotype. Int J Cancer 85: 176-181, 2000.

6. Kim MS, Lee EJ, Kim HR and Moon A: p38 kinase is a key signaling molecule for H-Ras-induced cell motility and invasive phenotype in human breast epithelial cells. Cancer Res 63: 5454-5461, 2003

7. Casey PJ, Solski PA, Der CJ and Buss JE: p21 ras is modified by a farnesyl isoprenoid. Proc Natl Acad Sci USA 86: 8323-8327, 1989.

8. Whyte DB, Kirschmeier P, Hockenberry TN, Nunez-Oliva I, James L, Catino JJ, Bishop WR and Pai JK: K- and N-Ras are geranylgeranylated in cells treated with farnesyl protein transferase inhibitors. J Biol Chem 272: 14459-14464, 1997.

9. Liao JK and Laufs U: Pleiotropic effects of statins. Annu Rev Pharmacol Toxicol 45: 89-118, 2005

10. Katz MS: Therapy insight: Potential of statins for cancer chemoprevention and therapy. Nat Clin Pract Oncol 2: 82-89, 2005.

11. Matar P, Rozados VR, Roggero EA and Scharovsky OG: Lovastatin inhibits tumor growth and metastasis development of a rat fibrosarcoma. Cancer Biother Radiopharm 13: 387-393, 1998.

12. Pedersen TR, Wilhelmsen L, Faergeman O, Strandberg TE, Thorgeirsson G, Troedsson L, Kristianson J, Berg K, Cook TJ, Haghfelt T, Kjekshus J, Miettinen T, Olsson AG, Pyörälä K and Wedel H: Follow-up study of patients randomized in the Scandinavian simvastatin survival study (4S) of cholesterol lowering. Am J Cardiol 86: 257-262, 2000. 
13. Fromigue O, Hamidouche $\mathrm{Z}$ and Marie PJ: Statin-induced inhibition of 3-hydroxy-3-methyl glutaryl coenzyme a reductase sensitizes human osteosarcoma cells to anticancer drugs. J Pharmacol Exp Ther 325: 595-600, 2008.

14. Moyad MA, Merrick GS, Butler WM, Wallner KE, Galbreath RW, Kurko B and Adamovich E: Statins, especially atorvastatin, may favorably influence clinical presentation and biochemical progression-free survival after brachytherapy for clinically localized prostate cancer. Urology 66: 1150-1154, 2005.

15. Collisson EA, Carranza DC, Chen IY and Kolodney MS: Isoprenylation is necessary for the full invasive potential of RhoA overexpression in human melanoma cells. J Invest Dermatol 119: 1172-1176, 2002.

16. Collisson EA, Kleer C, Wu M, De A, Gambhir SS, Merajver SD and Kolodney MS: Atorvastatin prevents RhoC isoprenylation, invasion, and metastasis in human melanoma cells. Mol Cancer Ther 2: 941-948, 2003.

17. Farina HG, Bublik DR, Alonso DF and Gomez DE: Lovastatin alters cytoskeleton organization and inhibits experimental metastasis of mammary carcinoma cells. Clin Exp Metastasis 19: 551-559, 2002

18. Denoyelle C, Vasse M, Korner M, Mishal Z, Ganné F, Vannier JP, Soria $\mathrm{J}$ and Soria C: Cerivastatin, an inhibitor of HMG-CoA reductase, inhibits the signaling pathways involved in the invasiveness and metastatic properties of highly invasive breast cancer cell lines: an in vitro study. Carcinogenesis 22: 1139-1148, 2001.

19. Furberg CD and Pitt B: Withdrawal of cerivastatin from the world market. Curr Control Trials Cardiovasc Med 2: 205-207, 2001.
20. Shin I, Kim S, Song H, Kim HR and Moon A: H-Ras-specific activation of Rac-MKK3/6-p38 pathway: its critical role in invasion and migration of breast epithelial cells. J Biol Chem 280: 14675-14683, 2005.

21. Endres M, Laufs U, Huang Z, Nakamura T, Huang $P$, Moskowitz MA and Liao JK: Stroke protection by 3-hydroxy-3methylglutaryl (HMG)-CoA reductase inhibitors mediated by endothelial nitric oxide synthase. Proc Natl Acad Sci USA 95: 8880-8885, 1998 .

22. Blauw GJ, Lagaay AM and Westendorp RG: Statins for prevention of stroke. Lancet 352: 144, 1998.

23. Fritz G: HMG-CoA reductase inhibitors (statins) as anticancer drugs (review). Int J Oncol 27: 1401-1409, 2005.

24. Campbell MJ, Esserman LJ, Zhou Y, Shoemaker M, Lobo M, Borman E, Baehner F, Kumar AS, Adduci K, Marx C, Petricoin EF, Liotta LA, Winters M, Benz S and Benz CC: Breast cancer growth prevention by statins. Cancer Res 66: 8707-8714, 2006.

25. Tryggvason K, Hoyhtya M and Pyke C: Type IV collagenases in invasive tumors. Breast Cancer Res Treat 24: 209-218, 1993.

26. Stetler-Stevenson WG, Brown PD, Onisto M, Levy AT and Liotta LA: Tissue inhibitor of metalloproteinases-2 (TIMP-2) mRNA expression in tumor cell lines and human tumor tissues. J Biol Chem 265: 13933-13938, 1990.

27. Turner NA, O'Regan DJ, Ball SG and Porter KE: Simvastatin inhibits MMP-9 secretion from human saphenous vein smooth muscle cells by inhibiting the RhoA/ROCK pathway and reducing MMP-9 mRNA levels. FASEB J 19: 804-806, 2005. 